\title{
STABILITY IN A BALL-PARTITION PROBLEM
}

\author{
THOMAS I. VOGEL
}

Received 16 August 2004

Stability for a capillary problem with surfaces meeting along a singular curve is analyzed using eigenvalue methods.

\section{Introduction}

In [4], stability of bubble clusters is characterized in terms of a quadratic form defined on the surfaces, which is then used to prove that the nonstandard double bubble is unstable. Since quadratic forms are often analyzed using eigenvalue methods, it seems natural to investigate the eigenvalue problem which arises from such a quadratic form. We will look at a specific example of a problem related to bubble clusters, in order to see how the quadratic form gives rise to interesting interactions across the singular curve.

The example that we will look at is the following. Suppose that we partition a ball into three regions of equal volume. How can this be done so that the sum of the areas of the partitioning surfaces is a minimum? We will not answer this general question, but we will address the stability of the particular partition given by three half-disks sharing the line segment $-1 \leq x \leq 1, y=0, z=0$ in their boundaries and meeting at angles of $(2 / 3) \pi$. It is clear that this configuration is stationary, since the contact angles between the free surfaces and the fixed sphere are all $\pi / 2$, and the behavior along the line segment satisfies the Plateau conditions (see [9]). This problem was chosen so that the various contributions due to curvature that appear in [4] would disappear, in that we could focus on the analysis rather than the geometry. It is important to realize that we cannot look at arbitrary normal perturbations on each surface. With the orientations of the normals that we will use, the condition that $f_{1}(p)+f_{2}(p)+f_{3}(p)=0$ must be imposed along the singular curve, so that infinitesimal perturbations will take the singular curve to a single curve rather than causing it to split. Following [4], we let $\mathscr{F}$ be the set of triples $\left\langle f_{1}, f_{2}, f_{3}\right\rangle$ with $f_{i} \in H^{1}\left(\sum_{i}\right)$ satisfying the matching condition $f_{1}+f_{2}+f_{3}=0$ along the singular curve $\gamma$.

We have an additional consideration in that we have terms in the derivatives relating to perturbations of bubble surfaces near fixed surfaces. These are handled in a manner similar to that used in [11], although there is a slight difference from that in [11], 
perturbations were assumed to be volume conserving across individual surfaces whereas we cannot make that assumption for the ball-partition problem. I am unaware of any work on partitioning balls into three volumes using a minimum of surface area. However, the analogous question with two volumes has been studied, see, for example, $[7,8]$.

\section{Results}

The problem that we're looking at has a fixed surface, so that it is not quite the same as the sorts of bubble clusters looked at in $[3,4]$. We must determine what additional terms come into consideration due to motion of the contact curve along the fixed surface. To see what happens to derivatives of surface area due to perturbations which move the contact curve with the fixed ball, consider a normal perturbation $\varepsilon f$ to the half-disk $\Sigma_{1}$, which we assume is contained in the upper half of the $x, y$ plane. We must subtract the area of the surface which goes outside of the ball. The situation is similar to that considered in [11], although it is much easier due to the special geometry. Putting the problem into cylindrical coordinates, we must subtract off the area of the surface $z=\varepsilon f(r \cos \theta, r \sin \theta)$ lying above the region $1-R(\theta, \varepsilon) \leq r \leq 1$, where $R(\theta, \varepsilon)$ is determined by

$$
(1-R)^{2}+\varepsilon^{2} f^{2}((1-R) \cos \theta,(1-R) \sin \theta)=1 .
$$

It is straightforward to verify by implicit differentiation that $R_{\varepsilon}(\theta, 0)=0$ and $R_{\varepsilon \varepsilon}(\theta, 0)=$ $-f^{2}(\cos \theta, \sin \theta)$. From this, it follows that we must subtract off the sum of the integrals of the squares of the perturbations along the contact curve of $\Sigma_{i}$ with the unit sphere from the quadratic form. With this, and plugging the specific curvatures of the surfaces and the singular curve into the formulas from [4], we have proven the following.

Proposition 2.1. The configuration for the ball-partitioning problem consisting of 3 halfdisks $\Sigma_{1}, \Sigma_{2}, \Sigma_{3}$ meeting along a diameter at an angle of $(2 / 3) \pi$ is stable if

$$
\sum_{i=1}^{3} \iint_{\Sigma_{i}}\left|\nabla f_{i}\right|^{2} d \Sigma-\int_{\sigma_{i}} f_{i}^{2} d \sigma \geq 0
$$

for every perturbation $\left\langle f_{1}, f_{2}, f_{3}\right\rangle \in \mathscr{F}$ for which

$$
\iint_{\Sigma_{1}} f_{1} d \Sigma=\iint_{\Sigma_{2}} f_{2} d \Sigma=\iint_{\Sigma_{3}} f_{3} d \Sigma
$$

that is, which infinitesimally preserves the volumes of the regions bounded by the $\sum_{i}$ 's. Here $\sigma_{i}$ is the semicircular part of the boundary of $\Sigma_{i}$.

Note 2.2. Similar calculations for perturbations of capillary surfaces which touch general fixed surfaces are performed in $[7,11]$. We cannot simply quote them here, however, since their calculations are for perturbations which keep the volume enclosed by a single surface fixed.

We now analyze the quadratic form in Proposition 2.1 as in $[5,6]$.

Theorem 2.3. The partition of the unit ball into three equal volumes by three half-disks meeting at $120^{\circ}$ is stable, that is, (2.2) holds on the subspace of F satisfying (2.3). 
Proof. We will first derive the eigenvalue problem for the quadratic form (2.2) in the absence of the volume constraint, and then we will find conditions for the quadratic form to be nonnegative on the subspace of $\mathscr{F}$ which satisfies (2.3). The interesting point of the eigenvalue problem is to see how the condition that solutions are in $\mathscr{F}$ is reflected in the boundary conditions along $\gamma$. Proceeding as in [2], the first eigenfunction $\left\langle\varphi_{0,1}, \varphi_{0,2}, \varphi_{0,3}\right\rangle$ will be the minimum of (2.2) subject to the constraint

$$
\sum_{i=1}^{3} \iint_{\Sigma_{i}} \varphi_{0, i}^{2} d \Sigma=1
$$

Let $\lambda_{0}$ be that minimum. Then

$$
\frac{\sum_{i=1}^{3} \iint_{\Sigma_{i}}\left|\nabla \varphi_{0, i}\right|^{2} d \Sigma-\int_{\sigma_{i}} \varphi_{0, i}^{2} d \sigma}{\sum_{i=1}^{3} \iint_{\Sigma_{i}} \varphi_{0, i}^{2} d \Sigma}=\lambda_{0} .
$$

Take an arbitrary triple $\left\langle\zeta_{1}, \zeta_{2}, \zeta_{3}\right\rangle$ in $\mathscr{F}$. We must have

$$
\sum_{i=1}^{3} \iint_{\Sigma_{i}}\left|\nabla\left(\varphi_{0, i}+\varepsilon \zeta_{i}\right)\right|^{2} d \Sigma-\int_{\sigma_{i}}\left(\varphi_{0, i}+\varepsilon \zeta_{i}\right)^{2} d \sigma \geq \lambda_{0}\left(\sum_{i=1}^{3} \iint_{\Sigma_{i}}\left(\varphi_{0, i}+\varepsilon \zeta_{i}\right)^{2} d \Sigma\right)
$$

holding for all $\varepsilon$. Multiplying out and using (2.5), we see that

$$
\begin{aligned}
& 2 \varepsilon\left(\sum_{i=1}^{3} \iint_{\Sigma_{i}} \nabla \zeta_{i} \cdot \nabla \varphi_{0, i} d \Sigma-\int_{\sigma_{i}} \varphi_{0, i} \zeta_{i} d \sigma-\lambda_{0} \iint_{\Sigma_{i}} \varphi_{0, i} \zeta_{i} d \Sigma\right) \\
& \quad+\varepsilon^{2}\left(\sum_{i=1}^{3} \iint_{\Sigma_{i}}\left|\nabla \zeta_{i}\right|^{2} d \Sigma-\int_{\sigma_{i}} \zeta_{i}^{2} d \sigma-\lambda_{0} \iint_{\Sigma_{i}} \zeta_{i}^{2} d \Sigma\right) \geq 0
\end{aligned}
$$

for all $\varepsilon$ and all admissible triples $\left\langle\zeta_{1}, \zeta_{2}, \zeta_{3}\right\rangle$. This can only be satisfied if the linear term in (2.7) is zero, that is,

$$
\sum_{i=1}^{3} \iint_{\Sigma_{i}} \nabla \zeta_{i} \cdot \nabla \varphi_{i, 0} d \Sigma-\int_{\sigma_{i}} \varphi_{i, 0} \zeta_{i} d \sigma=\lambda_{0} \iint_{\Sigma_{i}} \varphi_{i, 0} \zeta_{i} d \Sigma
$$

holds for all $\left\langle\zeta_{1}, \zeta_{2}, \zeta_{3}\right\rangle \in \mathscr{F}$.

Integrating by parts,

$$
\sum_{i=1}^{3}\left[\iint_{\Sigma_{i}} \zeta_{i}\left(-\Delta \varphi_{0, i}-\lambda_{0} \varphi_{0, i}\right) d \Sigma-\int_{\sigma_{i}} \zeta_{i}\left(\varphi_{0, i}-\frac{\partial \varphi_{0, i}}{\partial n}\right) d \sigma+\int_{\gamma} \zeta_{i} \frac{\partial \varphi_{0, i}}{\partial n} d \sigma\right]=0 .
$$

From this, we obtain the eigenvalue problem

$$
-\Delta \varphi_{0, i}=\lambda_{0} \varphi_{0, i}
$$

interior to $\Sigma_{i}$, with boundary conditions

$$
\frac{\partial \varphi_{0, i}}{\partial n}=\varphi_{0, i}
$$


1286 Stability in a ball-partition problem

on $\sigma_{i}$ and

$$
\frac{\partial \varphi_{0,1}}{\partial n}=\frac{\partial \varphi_{0,2}}{\partial n}=\frac{\partial \varphi_{0,3}}{\partial n}
$$

along the singular curve $\gamma$, along with the condition that

$$
\varphi_{0,1}+\varphi_{0,2}+\varphi_{0,3}=0
$$

holds on $\gamma$ ( since $\left.\left\langle\varphi_{0,1}, \varphi_{0,2}, \varphi_{0,3}\right\rangle \in \mathscr{F}\right)$.

The situation is similar enough to that considered in [1, Chapter 6], that the analogous results follow. Minimizing (2.2) with the additional constraint that $\left\langle\varphi_{1}, \varphi_{2}, \varphi_{3}\right\rangle$ be orthogonal to $\left\langle\varphi_{0,1}, \varphi_{0,2}, \varphi_{0,3}\right\rangle$ in $L^{2}\left(\Sigma_{1}\right) \oplus L^{2}\left(\Sigma_{2}\right) \oplus L^{2}\left(\Sigma_{3}\right)$ yields that eigenvector $\left\langle\varphi_{1,1}, \varphi_{1,2}, \varphi_{1,3}\right\rangle$ corresponding to the eigenvalue $\lambda_{1}$. Continuing, we obtain an orthonormal basis to $L^{2}\left(\Sigma_{1}\right) \oplus L^{2}\left(\Sigma_{2}\right) \oplus L^{2}\left(\Sigma_{3}\right)$ consisting of solutions to the eigenvalue problem

$$
-\Delta \varphi_{i}=\lambda \varphi_{i}
$$

with the same boundary conditions as before. Moreover,

$$
\lambda_{0} \leq \lambda_{1} \leq \cdots
$$

and $\lambda_{k} \rightarrow \infty$ as $k \rightarrow \infty$.

We must next determine all negative eigenvalues, along with their associated multiplicities. Identifying all $\Sigma_{i}$ 's as $\Sigma$, the unit upper half-disk in the $x, y$ plane, and $\sigma_{i}$ as $\sigma$, the unit upper half circle, we seek triples $\left\langle\varphi_{1}, \varphi_{2}, \varphi_{3}\right\rangle$ satisfying

$$
-\Delta \varphi_{i}=\lambda \varphi_{i}
$$

on $\Sigma$, with

$$
\frac{\partial \varphi_{i}}{\partial n}=\varphi_{i}
$$

on $\sigma$,

$$
\frac{\partial \varphi_{1}}{\partial n}=\frac{\partial \varphi_{2}}{\partial n}=\frac{\partial \varphi_{3}}{\partial n}
$$

on $\gamma$ (the line segment from $(-1,0)$ to $(1,0))$, and

$$
\varphi_{1}+\varphi_{2}+\varphi_{3}=0
$$

along $\gamma$.

Let $\psi=\varphi_{1}+\varphi_{2}+\varphi_{3}$. We must have

$$
-\Delta \psi=\lambda \psi
$$

on the half-disk, with

$$
\frac{\partial \psi}{\partial n}=\psi
$$


on $\sigma$ and

$$
\psi=0
$$

on $\gamma$. We may solve this by separation of variables. Writing $\psi$ as $R(r) \Psi(\theta)$, we must have $R^{\prime}(1)=R(1)$ and $\Psi(0)=\Psi(\pi)=0$. Substituting, we obtain

$$
R^{\prime \prime} \Psi+\frac{1}{r} R^{\prime} \Psi+\frac{1}{r^{2}} R \Psi^{\prime \prime}=-\lambda R \Psi
$$

hence

$$
\frac{R^{\prime \prime}}{R}+\frac{1}{r} \frac{R^{\prime}}{R}+\frac{1}{r^{2}} \frac{\Psi^{\prime \prime}}{\Psi}=-\lambda
$$

that is,

$$
r^{2} \frac{R^{\prime \prime}}{R}+r \frac{R^{\prime}}{R}+\lambda r^{2}=-\frac{\Psi^{\prime \prime}}{\Psi}=k
$$

for some separation constant $k$. The boundary conditions for $\Psi$ give that $\Psi(\theta)=c \sin (n \theta)$ for some integer $n \geq 1$. Since we seek a solution with negative $\lambda$, let $\lambda=-q^{2}$, yielding

$$
r^{2} R^{\prime \prime}+r R^{\prime}-\left(n^{2}+q^{2} r^{2}\right) R=0 .
$$

If $t=q r,(2.26)$ turns into the modified Bessel equation of order $n$ :

$$
t^{2} \frac{d^{2} R}{d t^{2}}+t \frac{d R}{d t}-\left(n^{2}+t^{2}\right) R=0
$$

The only solutions to (2.27) which are bounded at the origin are $c I_{n}(t)$, where $I_{n}$ is a modified Bessel function of the first kind (see [1]). Thus $R(r)=c I_{n}(q r)$. If $R^{\prime}(1)=R(1)$ is to be satisfied, there must be a positive integer $n$ and a $q>0$, so that

$$
q I_{n}^{\prime}(q)=I_{n}(q)
$$

However (again see [1]),

$$
I_{n}^{\prime}(q)=n \frac{I_{n}(q)}{q}+I_{n+1}(q)
$$

so that

$$
q I_{n}^{\prime}(q)-I_{n}(q)=(n-1) I_{n}(q)+q I_{n+1}(q)
$$

Since $n \geq 1$, then the right-hand side of $(2.30)$ is positive (since $I_{n}(q)$ is positive for positive $q$ ). This is a contradiction, since we want the left-hand side of the equation to be zero. Thus, the only possibility for $R(r)$ is that $c$ is zero, hence $\psi$ is identically zero. Thus $\varphi_{1}+\varphi_{2}+\varphi_{3}=0$ on the half-disk. From this, we see that

$$
\frac{\partial \varphi_{1}}{\partial n}+\frac{\partial \varphi_{2}}{\partial n}+\frac{\partial \varphi_{3}}{\partial n}=0
$$


on $\gamma$. Since the three normal derivatives are equal, it follows that

$$
\frac{\partial \varphi_{i}}{\partial n}=0
$$

on $\gamma$.

We now may find each of the $\varphi_{i}$ 's by separation of variables, using much the same reasoning as before. Write a generic $\varphi_{i}$ as

$$
\varphi=R(r) \Theta(\theta)
$$

the same equations hold as for $\psi$. The only difference is that the boundary conditions for $\Theta$ are that

$$
\Theta^{\prime}(0)=\Theta^{\prime}(\pi)
$$

making $\Theta(\theta)=\cos (n \theta)$ for some integer $n \geq 0$. This leads to $R(r)$ solving (2.26) and (2.27) as before, except that $n=0$ is now a possibility. From the previous argument, the only possibility for a solution yielding a negative eigenvalue is to have $n=0$, so that $\varphi$ is radially symmetric. For $n=0$, there will be precisely one solution $q_{0}$ to $(2.30)$, as it is not hard to show that $-I_{0}(q)+q I_{1}(q)$ is strictly increasing and changes sign. Numerically, $q_{0}$ is approximately 1.6083 , leading to the negative eigenvalue -2.5866 . The eigenspace in $\mathscr{F}$ corresponding to this eigenvalue is two dimensional: it is

$$
\left\langle a_{1} I_{0}\left(q_{0} r\right), a_{2} I_{0}\left(q_{0} r\right), a_{3} I_{0}\left(q_{0} r\right)\right\rangle,
$$

where

$$
a_{1}+a_{2}+a_{3}=0
$$

is required for the triple to be in $\mathscr{F}$. Thus there is a single negative eigenvalue, of multiplicity two.

Certainly, therefore, the quadratic form (2.2) is not nonnegative on all of $\mathscr{F}$. To see whether it is nonnegative on the subspace of $\mathscr{F}$ satisfying (2.3), we follow the approach of $[5,6,10]$. Since the subspace satisfying the volume constraint is the set of vectors in $L^{2}(\Sigma)^{3}$ orthogonal to $\langle 1,-1,0\rangle$ and $\langle 1,0,-1\rangle$, we first seek $\vec{\eta}=\left\langle\eta_{1}, \eta_{2}, \eta_{3}\right\rangle$ and $\vec{\zeta}=\left\langle\zeta_{1}, \zeta_{2}, \zeta_{3}\right\rangle$ satisfying

$$
\begin{aligned}
& -\Delta \vec{\eta}=\langle 1,-1,0\rangle \\
& -\Delta \vec{\zeta}=\langle 1,0,-1\rangle
\end{aligned}
$$

with boundary conditions as in (2.11), (2.12), and (2.13).

Specific radially symmetric solutions are given by

$$
\begin{aligned}
& \vec{\eta}=\left\langle-\frac{1}{4} r^{2}-\frac{1}{4}, \frac{1}{4} r^{2}+\frac{1}{4}, 0\right\rangle, \\
& \vec{\zeta}=\left\langle-\frac{1}{4} r^{2}-\frac{1}{4}, 0, \frac{1}{4} r^{2}+\frac{1}{4}\right\rangle .
\end{aligned}
$$


The condition that we must check is whether

$$
\left(\begin{array}{ll}
(\vec{\eta},-\Delta \vec{\eta}) & (\vec{\eta},-\Delta \vec{\zeta}) \\
(\vec{\zeta},-\Delta \vec{\eta}) & (\vec{\zeta},-\Delta \vec{\zeta})
\end{array}\right)
$$

is negative definite, where each entry is an inner product in $L^{2}(\Sigma)^{3}$. It is easy to verify that this is true.

Having done that, we outline the rest of the argument. Given a perturbation $\vec{f}=$ $\left\langle f_{1}, f_{2}, f_{3}\right\rangle$ which satisfies (2.3), that is, which is orthogonal to $\langle 1,-1,0\rangle$ and $\langle 1,0,-1\rangle$ in $L^{2}(\Sigma)^{3}$, one may show that there exist $c_{1}$ and $c_{2}$ so that $\vec{f}+c_{1} \vec{\eta}+c_{2} \vec{\zeta}$ is orthogonal to the two-dimensional eigenspace corresponding to the negative eigenvalue. The fact that (2.39) is negative definite is used for this. Calling $\vec{f}+c_{1} \vec{\eta}+c_{2} \vec{\zeta} \vec{g}$, one can verify that

$$
(\vec{f},-\Delta \vec{f})=(\vec{g},-\Delta \vec{g})-\left[\begin{array}{ll}
c_{1} & c_{2}
\end{array}\right]\left(\begin{array}{ll}
(\vec{\eta},-\Delta \vec{\eta}) & (\vec{\eta},-\Delta \vec{\zeta}) \\
(\vec{\zeta},-\Delta \vec{\eta}) & (\vec{\zeta},-\Delta \vec{\zeta})
\end{array}\right)\left[\begin{array}{l}
c_{1} \\
c_{2}
\end{array}\right] \geq 0
$$

again using (2.39), concluding the proof. For more details, see one of the three references mentioned.

Note 2.4. The eigenvalue approach used in this paper can be applied to the sorts of problems considered in [4]. This is more a matter of taste than anything: the results will be equivalent to those found using Jacobi fields as in [4].

\section{References}

[1] M. Abramowitz and I. A. Stegun, eds., Handbook of Mathematical Functions, with Formulas, Graphs, and Mathematical Tables, National Bureau of Standards Applied Mathematics Series, vol. 55, Superintendent of Documents, U.S. Government Printing Office, District of Columbia, 1965, 3rd printing, with corrections.

[2] R. Courant and D. Hilbert, Methods of Mathematical Physics. Vol. I, Interscience Publishers, New York, 1953.

[3] J. Hass and R. Schlafly, Double bubbles minimize, Ann. of Math. (2) 151 (2000), no. 2, 459-515.

[4] M. Hutchings, F. Morgan, M. Ritoré, and A. Ros, Proof of the double bubble conjecture, Ann. of Math. (2) 155 (2002), no. 2, 459-489.

[5] J. H. Maddocks, Restricted quadratic forms and their application to bifurcation and stability in constrained variational principles, SIAM J. Math. Anal. 16 (1985), no. 1, 47-68.

[6] Stability and folds, Arch. Ration. Mech. Anal. 99 (1987), no. 4, 301-328.

[7] A. Ros and R. Souam, On stability of capillary surfaces in a ball, Pacific J. Math. 178 (1997), no. 2, 345-361.

[8] A. Ros and E. Vergasta, Stability for hypersurfaces of constant mean curvature with free boundary, Geom. Dedicata 56 (1995), no. 1, 19-33.

[9] J. E. Taylor, The structure of singularities in soap-bubble-like and soap-film-like minimal surfaces, Ann. of Math. 103 (1976), no. 3, 489-539. 
1290 Stability in a ball-partition problem

[10] T. I. Vogel, Sufficient conditions for multiply constrained extrema, Pacific J. Math. 180 (1997), no. 2, 377-383.

[11] H. C. Wente, Existence theorems for surfaces of constant mean curvature and perturbations of a liquid globule in equilibrium, Ph.D. thesis, Harvard University, Massachusetts, 1966.

Thomas I. Vogel: Department of Mathematics, Texas A\&M University, College Station, TX 77843, USA

E-mail address: tvogel@math.tamu.edu

Current address: Max Planck Institute for Mathematics in the Sciences, Inselstraße 22, 04103 Leipzig, Germany 


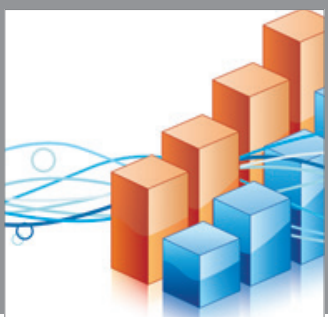

Advances in

Operations Research

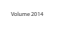

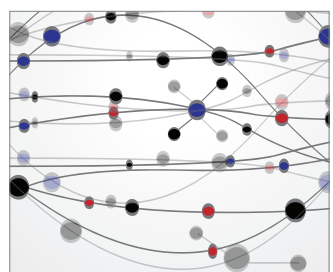

\section{The Scientific} World Journal
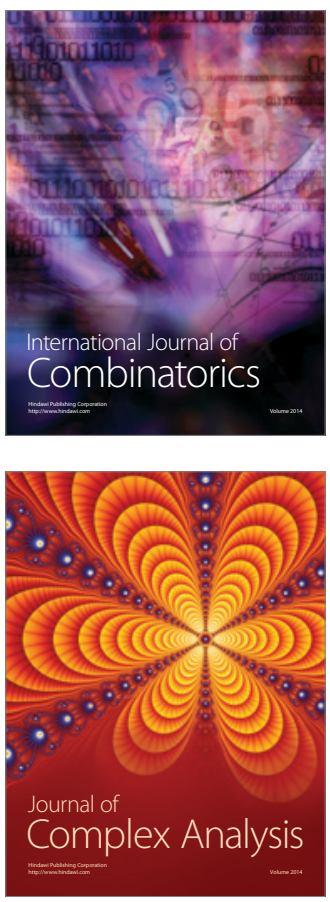

International Journal of

Mathematics and

Mathematical

Sciences
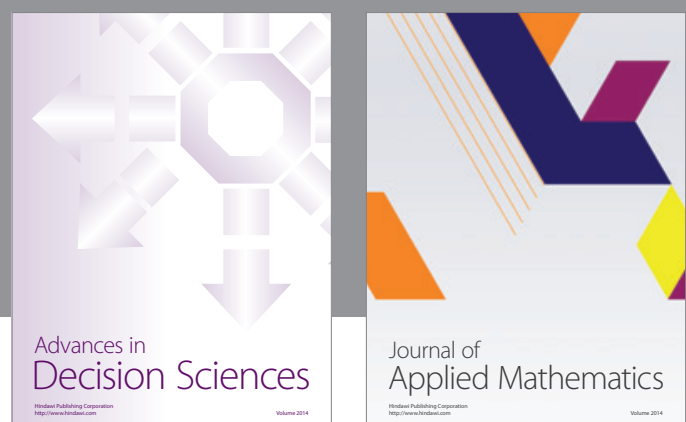

Journal of

Applied Mathematics
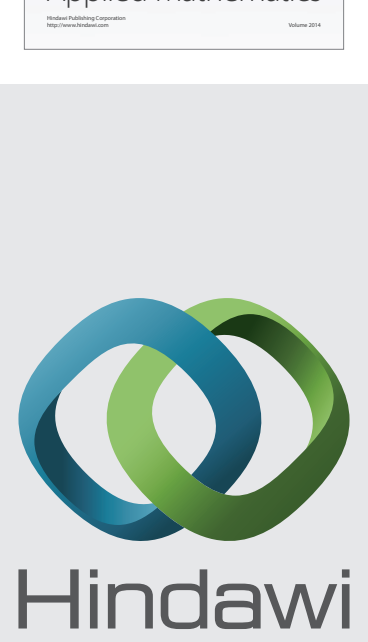

Submit your manuscripts at http://www.hindawi.com
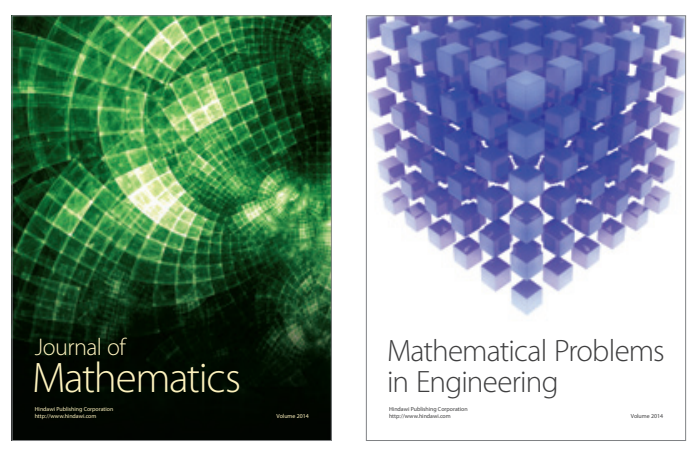

Mathematical Problems in Engineering
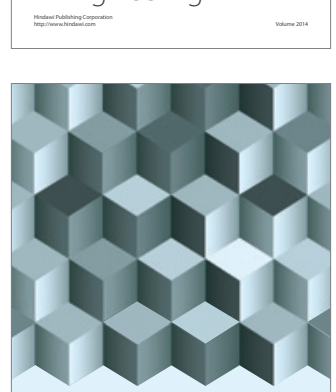

Journal of

Function Spaces
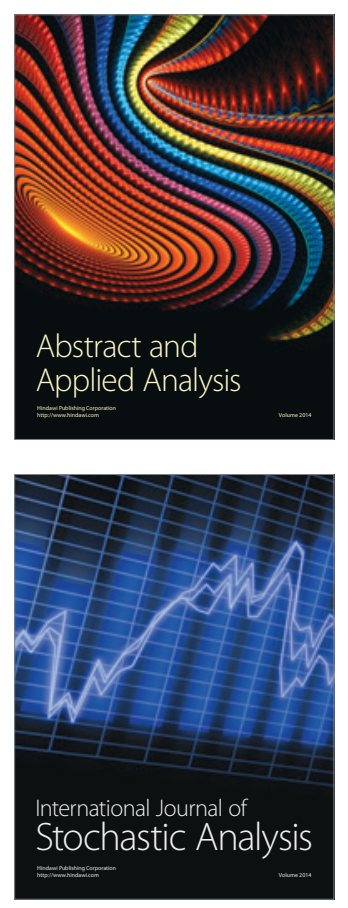

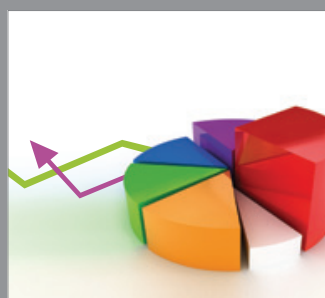

ournal of

Probability and Statistics

Promensencen
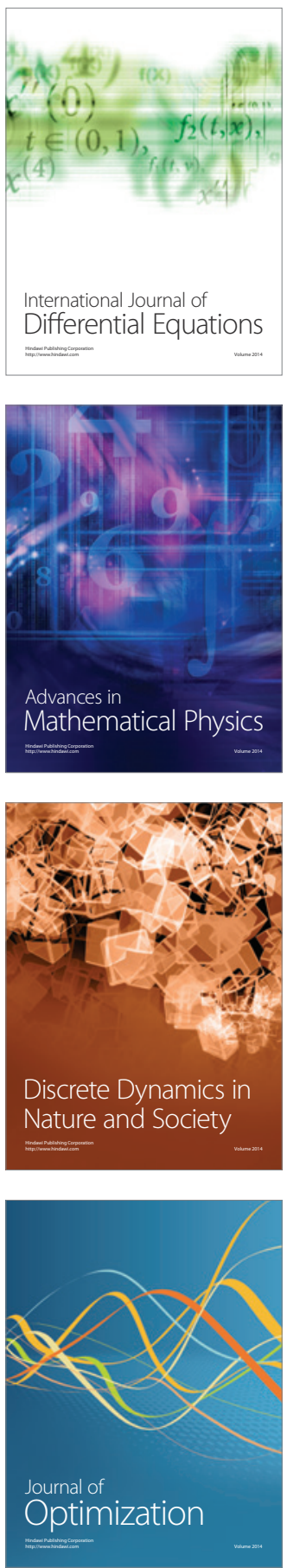